\title{
Lower bounds on the obstacle number of graphs *
}

\author{
Padmini Mukkamala \\ McDaniel College \\ Budapest, Hungary \\ padmini.mvs@gmail.com \\ János Pach \\ Ecole Polytechnique Fédérale de Lausanne \\ Lausanne, Switzerland \\ pach@cims.nyu.edu \\ Dömötör Pálvölgyi \\ Eötvös University \\ Budapest, Hungary \\ dom@cs.elte.hu
}

Submitted: Mar 9, 2011; Accepted: May 23, 2012; Published: Jun 6, 2012

Mathematics Subject Classifications: 05C62, 68R10

\begin{abstract}
Given a graph $G$, an obstacle representation of $G$ is a set of points in the plane representing the vertices of $G$, together with a set of connected obstacles such that two vertices of $G$ are joined by an edge if and only if the corresponding points can be connected by a segment which avoids all obstacles. The obstacle number of $G$ is the minimum number of obstacles in an obstacle representation of $G$. It is shown that there are graphs on $n$ vertices with obstacle number at least $\Omega(n / \log n)$.
\end{abstract}

Keywords: Obstacle number, visibility graph, graph representation

${ }^{*}$ The authors gratefully acknowledge support from the Bernoulli Center at EPFL and from the Swiss National Science Foundation, Grant No. 200021-125287/1. Research by the second author was also supported by grants from NSF, NSA, and OTKA. Research by the second and third author was also supported by EUROGIGA project GraDR 10-EuroGIGA-OP-003 (OTKA NN 102029). 


\section{Introduction}

Consider a set $P$ of points in the plane and a set of closed polygonal obstacles whose vertices together with the points in $P$ are in general position, that is, no three of them are on a line. The corresponding visibility graph has $P$ as its vertex set, two points $p, q \in P$ being connected by an edge if and only if the segment $p q$ does not meet any of the obstacles. Visibility graphs are extensively studied and used in computational geometry, robot motion planning, computer vision, sensor networks, etc.; see [4], [7], [12], [13], [21].

Alpert, Koch, and Laison [2] introduced an interesting new parameter of graphs, closely related to visibility graphs. Given a graph $G$, we say that a set of points and a set of polygonal obstacles as above constitute an obstacle representation of $G$, if the corresponding visibility graph is isomorphic to $G$. A representation with $h$ obstacles is also called an $h$-obstacle representation. The smallest number of obstacles in an obstacle representation of $G$ is called the obstacle number of $G$ and is denoted by obs $(G)$. Alpert et al. [2] proved that there exist graphs with arbitrarily large obstacle numbers.

Using tools from extremal graph theory, it was shown in [16] that for any fixed $h$, the number of graphs with obstacle number at most $h$ is $2^{o\left(n^{2}\right)}$. Notice that this immediately implies the existence of graphs with arbitrarily large obstacle numbers.

In the present note, we establish some more precise estimates.

Theorem 1. (i) For any positive integer $h$, the number of graphs on $n$ (labeled) vertices that admit a representation with $h$ obstacles is at most

$$
2^{O\left(h n \log ^{2} n\right)}
$$

(ii) Moreover, the number of graphs on $n$ (labeled) vertices that admit a representation with a set of obstacles having a total of sides, is at most

$$
2^{O(n \log n+\operatorname{sog} s)} .
$$

In the above bounds, it makes no difference whether we count labeled or unlabeled graphs, because the number of labeled graphs is at most $n !=2^{O(n \log n)}$ times the number of unlabeled ones.

It follows from Theorem 1 (i) that for every $n$, there exists a graph $G$ on $n$ vertices with obstacle number

$$
\operatorname{obs}(G) \geqslant \Omega\left(n / \log ^{2} n\right) .
$$

Indeed, as long as $2^{O\left(h n \log ^{2} n\right)}$ is smaller than $2^{\Omega\left(n^{2}\right)}$, the total number of (labeled) graphs with $n$ vertices, we can find at least one graph on $n$ vertices with obstacle number $h$.

Here we show the following slightly stronger bound.

Theorem 2. For every $n$, there exists a graph $G$ on $n$ vertices with obstacle number

$$
\operatorname{obs}(G) \geqslant \Omega(n / \log n)
$$


This comes close to answering the question in [2] whether the obstacle number of every graph with $n$ vertices is at most $O(n)$. However, we have no upper bound on the maximum obstacle number of $n$-vertex graphs, better than $O\left(n^{2}\right)$. In fact, we conjecture that the truth is somewhere around $n^{2}$.

Our next theorem answers another question from [2].

Theorem 3. For every $h$, there exists a graph with obstacle number exactly $h$.

A special instance of the obstacle problem has received a lot of attention, due to its connection to the Szemerédi-Trotter theorem on incidences between points and lines [20], [19], and other classical problems in incidence geometry [15]. This is to decide whether the obstacle number of $\bar{K}_{n}$, the empty graph on $n$ vertices, is $O(n)$ if the obstacles must be points. This question is commonly called "the blocking conjecture"; for related problems, see the survey by Pór and Wood [17]. The best known upper bound is $n 2^{O(\sqrt{\log n})}$ is due to Pach [14] (see also Dumitrescu et al. [5], Matoušek [10], Aloupis et al. [1], and Stanchescu [18] for a related result), while a superlinear lower bound is conjectured by most experts.

It is an interesting open problem to decide whether the obstacle number of planar graphs can be bounded from above by a constant. For outerplanar graphs, one obstacle is enough, as shown in [2]. Fulek, Saeedi, and Sarı̈z [6] have also proved that every outerplanar graph has a representation with at most five convex obstacles.

Theorem $i$ is proved in Section $i+1,1 \leqslant i \leqslant 3$.

\section{Proof of Theorem 1}

We will prove the theorem by a simple counting method. Before turning to the proof, we introduce some terminology. Given any placement (embedding) of the vertices of $G$ in general position in the plane, a straight-line drawing or, in short, a drawing of $G$ consists of the image of the embedding and the set of open line segments connecting all pairs of points that correspond to the edges of $G$. If there is no danger of confusion, we make no notational difference between the vertices of $G$ and the corresponding points, and between the pairs $u v$ and the corresponding open segments. The complement of the set of all points that correspond to a vertex or belong to at least one edge of $G$ falls into connected components. These components are called the faces of the drawing. Notice that if $G$ has an obstacle representation with a particular placement of its vertex set, then

(1) each obstacle must lie entirely in one face of the drawing, and

(2) each non-edge of $G$ must be blocked by at least one of the obstacles.

We start by proving a result about the convex obstacle number (a special case of Theorem 2), as the arguments are simpler here. Then we tackle Theorem 1 using similar methods.

Following Alpert et al., we define the convex obstacle number $\operatorname{obs}_{c}(G)$ of a graph $G$ as the minimal number of obstacles in an obstacle representation of $G$, in which each obstacle is convex.

Claim 4. For every $n$, there exists a graph $G$ on $n$ vertices with convex obstacle number

$$
\operatorname{obs}_{c}(G) \geqslant \Omega(n / \log n) \text {. }
$$


The idea is to find a short encoding of the obstacle representations of graphs, and to use this to give an upper bound on the number of graphs with low obstacle number. The proof uses the concept of order types. Two sets of points, $P_{1}$ and $P_{2}$, in general position in the plane are said to have the same order type if there is a one to one correspondence between them with the property that the orientation of any triple in $P_{1}$ is the same as the orientation of the corresponding triple in $P_{2}$. Counting the number of different order types is a classical task.

Theorem A. [Goodman, Pollack [8]] The number of different order types of $n$ points in general position in the plane is $2^{O(n \log n)}$.

Observe that asymptotically the same upper bound holds for the number of different order types of $n$ labeled points, because the number of different permutations of $n$ points is $n !=2^{O(n \log n)}$.

Proof of Claim 4 We will give an upper bound for the number of graphs that admit a representation with at most $h$ convex obstacles. Let us fix such a graph $G$, together with a representation. Let $V$ be the set of points representing the vertices, and let $O_{1}, \ldots, O_{h}$ be the convex obstacles. For any obstacle $O_{i}$, rotate an oriented tangent line $\ell$ along its boundary in the clockwise direction. We can assume without loss of generality that $\ell$ never passes through two points of $V$. Let us record the sequence of points met by $\ell$. If $v \in V$ is met at the right side of $\ell$, we add the symbol $v^{+}$to the sequence, otherwise we add $v^{-}$. (See Figure 1.)

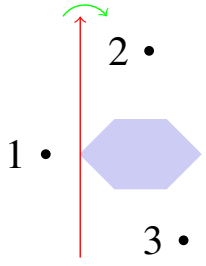

(a) Empty

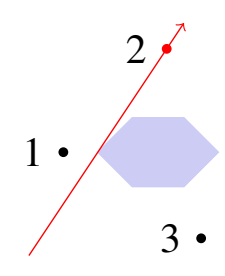

(b) $2^{+}$

$2 \cdot$

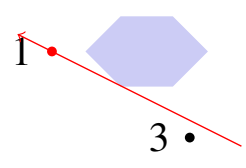

(f) $2^{+} 1^{-} 2^{-} 3^{+} 1^{+}$

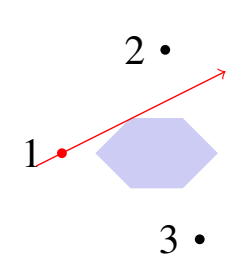

(c) $2^{+} 1^{-}$

2 •

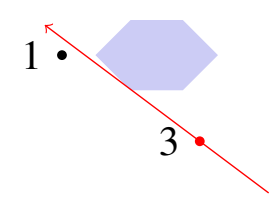

(g)

$2^{+} 1^{-} 2^{-} 3^{+} 1^{+} 3^{-}$

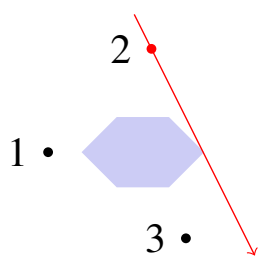

(d) $2^{+} 1^{-} 2^{-}$

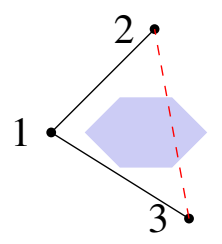

(h) $2^{+} 1^{-} 2^{-} 3^{+} 1^{+} 3^{-}$

Figure 1: Parts (a) to (g) show the construction of the sequence and (h) shows the visibilities. The arrow on the tangent line indicates the direction from the point of tangency in which we assign + as a label to the vertex. The additional arrow in (a) indicates that the tangent line is rotated clockwise around the obstacle.

When $\ell$ returns to its initial position, we stop. The resulting sequence consists of $2 n$ characters. From this sequence, it is easy to reconstruct which pairs of vertices are visible in the presence of the single obstacle $O_{i}$. Hence, knowing these sequences for every obstacle $O_{i}$, completely 
determines the visibility graph $G$. The number of distinct sequences assigned to a single obstacle is at most $(2 n)$ !, so the number of graphs with convex obstacle number at most $h$ cannot exceed

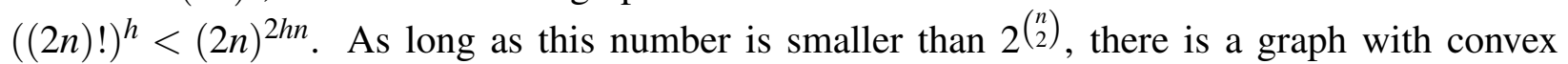
obstacle number larger than $h$.

To prove Theorem[1, we will need one more result. Given a drawing of a graph, the complexity of a face is the number of line-segment sides bordering it. The following result was proved by Arkin, Halperin, Kedem, Mitchell, and Naor (see Matoušek, Valtr [9] for its sharpness).

Theorem B. [Arkin et al. [3]] The complexity of a single face in a drawing of a graph with $n$ vertices is at most $O(n \log n)$.

Note that this bound does not depend on the number of edges of the graph.

Proof of Theorem 1. First we show how to reduce part (i) of the theorem to part (ii). For each graph $G$ with $n$ vertices that admits a representation with at most $h$ obstacles, fix such a representation. Consider the visibility graph $G$ of the vertices in this representation. As explained at the beginning of this section, each obstacle belongs to a single face in this drawing. In view of Theorem $\mathrm{B}$, the complexity of every face is $O(n \log n)$. Replacing each obstacle by a slightly shrunken copy of the face containing it, we can achieve that every obstacle $i s$ a polygonal region with $O(n \log n)$ sides. We have $\log (h n \log n)=O(\log n)$, since $h \leqslant\left(\begin{array}{l}n \\ 2\end{array}\right)$, so we are done.

Next we prove part (ii). Notice that the order type of the sequence $S$ starting with the vertices of $G$, followed by the vertices of the obstacles (listed one by one, in cyclic order, and properly separated from one another), completely determines $G$. That is, we have a sequence of length $N$ with $N \leqslant n+s$. According to Theorem A (and the comment following it), the number of different order types with this many points is at most

$$
2^{O(N \log N)}<2^{c(n+s) \log (n+s)}
$$

for a suitable constant $c>0$. This is a very generous upper bound: most of the above sequences do not correspond to any visibility graph $G$.

Following Alpert et al., we define the segment obstacle number $\operatorname{obs}_{s}(G)$ of a graph $G$ as the minimal number of obstacles in an obstacle representation of $G$, in which each obstacle is a (straight-line) segment. If we only allow segment obstacles, we have $s=2 n$, and thus Theorem 1 (ii) implies the following bound.

Corollary 5. For every $n$, there exists a graph $G$ on $n$ vertices with segment obstacle number

$$
\operatorname{obs}_{s}(G) \geqslant \Omega\left(n^{2} / \log n\right) \text {. }
$$

In general, as long as the sum of the sides of the obstacles, $s$, satisfies $s \log s=o\left(\left(\begin{array}{l}n \\ 2\end{array}\right)\right)$, we can argue that there is a graph that cannot be represented with such obstacles. 


\section{Proof of Theorem 2}

Before turning to the proof, we need a simple property of obstacle representations.

Lemma 6. Let $k>0$ be an integer and let $G$ be a graph with $n$ vertices that has an obstacle representation with fewer than $\frac{n}{2 k}$ obstacles. Then $G$ has at least $\left\lfloor\frac{n}{2 k}\right\rfloor$ vertex disjoint induced subgraphs of $k$ vertices with obstacle number at most one.

Proof. Fix an obstacle representation of $G$ with fewer than $\frac{n}{2 k}$ obstacles. Suppose without loss of generality that in this representation no two vertices have the same $x$-coordinate. Using vertical lines, divide the vertices of $G$ into $\left\lfloor\frac{n}{k}\right\rfloor$ groups of size $k$ and possibly a last group that contains fewer than $k$ vertices. Let $G_{1}, G_{2}, \ldots$ denote the subgraphs of $G$ induced by these groups. Notice that if the convex hull of the vertices of $G_{i}$ does not entirely contain an obstacle, then obs $\left(G_{i}\right) \leqslant 1$. Therefore, the number of subgraphs $G_{i}$ that have $k$ points and obstacle number at most one is larger than $\left\lfloor\frac{n}{k}\right\rfloor-\frac{n}{2 k}$, and the lemma is true.

We prove Theorem 2 by a probabilistic argument.

Proof of Theorem 2. Let $G$ be a random graph on $n$ labeled vertices, whose edges are chosen independently with probability $1 / 2$. Let $k$ be a positive integer to be specified later. According to Lemma 6 .

$$
\operatorname{Prob}[\operatorname{obs}(G)<n /(2 k)]
$$

can be estimated from above by the probability that $G$ has at least $\left\lfloor\frac{n}{2 k}\right\rfloor$ vertex disjoint induced subgraphs of $k$ vertices such that each of them has obstacle number at most one. Let $p(n, k)$ denote the probability that $G$ satisfies this latter condition.

Suppose that $G$ has $\lfloor n /(2 k)\rfloor$ vertex disjoint induced subgraphs $G_{1}, G_{2}, \ldots$ with $\left|V\left(G_{i}\right)\right|=k$ and obs $\left(G_{i}\right) \leqslant 1$. The vertices of $G_{1}, G_{2}, \ldots$ can be chosen in at most $\left(\begin{array}{l}n \\ k\end{array}\right)^{\lfloor n /(2 k)\rfloor}$ different ways. It follows from Theorem 1 (i) that the probability that a fixed $k$-tuple of vertices in $G$ induces a subgraph with obstacle number at most one is at most

$$
2^{O\left(k \log ^{2} k\right)-\left(\begin{array}{l}
k \\
2
\end{array}\right)}
$$

For disjoint $k$-tuples of vertices, the events that the obstacle numbers of their induced subgraphs do not exceed one are independent.

Therefore, we have

$$
p(n, k) \leqslant\left(\begin{array}{l}
n \\
k
\end{array}\right)^{\lfloor n /(2 k)\rfloor} \cdot\left(2^{\left.O\left(k \log ^{2} k\right)-\left(\begin{array}{l}
k \\
2
\end{array}\right)\right)^{\lfloor n /(2 k)\rfloor} \leqslant 2^{n \log n-n k / 4+O\left(n \log ^{2} k\right)} .}\right.
$$

Setting $k=\lfloor 5 \log n\rfloor)$, the right-hand side of the last inequality tends to zero. In this case, almost all graphs on $n$ vertices have obstacle number at least $\frac{n}{2 k}>\frac{n}{10 \log n}$, which completes the proof. 


\section{Proof of Theorem 3}

Alpert, Koch, and Laison [2] asked whether for every natural number $h$ there exists a graph whose obstacle number is exactly $h$. Here we answer this question in the affirmative.

Proof of Theorem 3. Pick a graph $G$ with obstacle number $h^{\prime}>h$. (The existence of such a graph was first proved in [2], but it also follows from Theorem 2, ) Let $n$ denote the number of vertices of $G$. Consider the complete graph $K_{n}$ on $V(G)$. Clearly, obs $\left(K_{n}\right)=0$, and $G$ can be obtained from $K_{n}$ by successively deleting edges. Observe that as we delete an edge from a graph $G^{\prime}$, its obstacle number cannot increase by more than one. Indeed, if we block the deleted edge $e$ by adding a very small obstacle that does not intersect any other edge of $G^{\prime}$, we obtain a valid obstacle representation of $G^{\prime}-e$. (Of course, the obstacle number of a graph can also decrease by the removal of an edge.) At the beginning of the process, $K_{n}$ has obstacle number zero, at the end $G$ has obstacle number $h^{\prime}>h$, and whenever it increases, the increase is one. We can conclude that at some stage we obtain a graph with obstacle number precisely $h$.

The same argument applies to the convex obstacle number, to the segment obstacle number, and many similar parameters.

\section{Acknowledgement.}

We are indebted to Deniz Sarı̈z for many valuable discussions on the subject. We would also like to thank the anonymous referees for their comments. A conference version containing some results from this paper and some from [16] appeared in [11].

\section{References}

[1] Greg Aloupis, Brad Ballinger, Sebastien Collette, Stefan Langerman, Attila Pór, and David R. Wood. Blocking coloured point sets. In János Pach, editor, Thirty Essays in Geometric Graph Theory, Algorithms and Combinatorics Series. Springer, 2012.

[2] Hannah Alpert, Christina Koch, and Joshua D. Laison. Obstacle numbers of graphs. Discrete \& Computational Geometry, 44(1):223-244, 2010.

[3] Esther M. Arkin, Dan Halperin, Klara Kedem, Joseph S. B. Mitchell, and Nir Naor. Arrangements of segments that share endpoints: single face results. Discrete \& Computational Geometry, 13(3-4):257-270, 1995.

[4] Mark de Berg, Marc van Kreveld, Mark Overmars, and Otfried Schwarzkopf. Computational Geometry. Algorithms and Applications (2nd ed.). Springer-Verlag, Berlin, 2000.

[5] Adrian Dumitrescu, János Pach, and Géza Tóth. A note on blocking visibility between points. Geombinatorics, 19(1):67-73, 2009.

[6] Radoslav Fulek, Deniz Sarı̈z, and Noushin Saeedi. Convex obstacle number of outerplanar graphs and bipartite permutation graphs. In János Pach, editor, Thirty Essays in Geometric Graph Theory, Algorithms and Combinatorics Series. Springer, 2012. 
[7] Subir Kumar Ghosh. Visibility algorithms in the plane. Cambridge University Press, Cambridge, 2007.

[8] Jacob E. Goodman and Richard Pollack. Upper bounds for configurations and polytopes in $\mathbf{R}^{d}$. Discrete Comput. Geom., 1(3):219-227, 1986.

[9] Jirí Matoušek and Pavel Valtr. The complexity of lower envelope of segments with $h$ endpoints. Intuitive Geometry, Bolyai Society of Math. Studies, 6:407-411, 1997.

[10] Jiří Matoušek. Blocking visibility for points in general position. Discrete \& Computational Geometry, 42(2):219-223, 2009.

[11] Padmini Mukkamala, János Pach, and Deniz Sariöz. Graphs with large obstacle numbers. In Dimitrios M. Thilikos, editor, WG, volume 6410 of Lecture Notes in Computer Science, pages 292-303, 2010.

[12] Joseph O'Rourke. Visibility. In Handbook of discrete and computational geometry, CRC Press Ser. Discrete Math. Appl., pages 467-479. CRC, Boca Raton, FL, 1997.

[13] Joseph O'Rourke. Open problems in the combinatorics of visibility and illumination. In Advances in discrete and computational geometry (South Hadley, MA, 1996), volume 223 of Contemp. Math., pages 237-243. Amer. Math. Soc., Providence, RI, 1999.

[14] János Pach. Midpoints of segments induced by a point set. Geombinatorics, 13(2):98-105, 2003.

[15] János Pach and Pankaj K. Agarwal. Combinatorial geometry. Wiley-Interscience Series in Discrete Mathematics and Optimization. John Wiley \& Sons Inc., New York, 1995. A Wiley-Interscience Publication.

[16] János Pach and Deniz Sariöz. On the structure of graphs with low obstacle number. Graphs and Combinatorics, 27(3):465-473, 2011.

[17] Attila Pór and David R. Wood. On visibility and blockers. J. Comput. Geom., 1(1):29-40, 2010.

[18] Yonutz V. Stanchescu. Planar sets containing no three collinear points and non-averaging sets of integers. Discrete Math., 256(1-2):387-395, September 2002.

[19] Endre Szemerédi and William T. Trotter, Jr. A combinatorial distinction between the Euclidean and projective planes. European J. Combin., 4(4):385-394, 1983.

[20] Endre Szemerédi and William T. Trotter, Jr. Extremal problems in discrete geometry. Combinatorica, 3(3-4):381-392, 1983.

[21] Jorge Urrutia. Art gallery and illumination problems. In Handbook of computational geometry, pages 973-1027. North-Holland, Amsterdam, 2000. 